

\section{Improving Scintillation Crystals Using Muon Tomography ${ }^{\dagger}$ *}

D. H. Dowe11, B. J. Flneman, and A. M. Sandorf1

Fhystcs Department, Brookhaven National Laboratory, Upton, New York, 11973

\section{Introduction}

A new high-energy $\gamma$-ray spectrometer is belng bullt for the LaserElectron-Gamma Source (LEGS) at Brookhaven ${ }^{\perp}$. This spectrometer will measure up to $400 \mathrm{MeV} Y$ rays with good energy resolution and high efficiency. The required energy resolution is determined by the necessity to separate elastic and Inelastic photon scattering as well as coherent and incoherent neutral plon production. This means that an energy resolution of a fev MeV or approximately $1 \%$ to $2 \%$ is needed. Also, since the count rates are very low (tens of counts per hour), the detection efficlency should be no less than $50 \%$ of the geometric solid angle, resulting in an ex .. ency-solid angle product of about 100 msr.

It is this desire to measure $\gamma$-ray spectra with good energy resolution and efflciency which motivates our study of NaI(T1) detectors. of the scintillation media to choose from, $\mathrm{NaI}(\mathrm{T} 1)$ is the most developed and 18 avallable In large single ingots of high quality. However, for the most part previously built NaI(TI) spectrometers have been limlted to resolutiong from $4 \%$ to $10 \%$ in this energy range. This poor performance of NaI(TI) is caused by two major

This work has been performed under contract DE-AC02-76CH00016 with the United States Department of Energy.

*Presented at "Workshop on Photon and Neutral Meson Physics", Los Alamos National Laboratory, January 7-9, 1987 
effects, detector size and non-uniform light generation and collection. We have studied the resolution and efficiency effects due to detector size from 50 to $300 \mathrm{MeV}$ and are building a detector $48.3 \mathrm{ctm}$ dianeter by $48.3 \mathrm{~cm} 1$ long from a single, unsegmented crystal ${ }^{2}$. The second problem of uniformity is the topic of this paper.

We will first briefly describe the currently used method of surface compensation with low energy p-ray sources. This method cannot probe the interior region of a $\mathrm{NaI}(\mathrm{T} 1)$ crystal larger than about $20 \mathrm{~cm}$ to $25 \mathrm{~cm}$ in diameter, since low energy sources penetrate only a shell near the surface. A new technique is then described which uses high energy muons to probe the full crystal volume, and reconstruct an Image of the Interfor using Computer Alded Tomography (CAT) methods.

II. Current Surface Compensation Techniques

At high $\gamma$-ray energies the resolution of a sufficiently large NaI(Tl) crystal is limited by the nonuniformity of light collection and by variations in the concentration of the Thallium dopant as well as crystal dislocations and impurities. The light collection from different regions of a large single crystal will change smoothly due to solld angle effects. (Abrupt changes will be evident in segmented detectors at the optical foints.) on the other hand, the Thallium and/or Impurity concentration variations will produce varying light output which is strongly dependent upon location in the detector. Hence the same energy deposition in different portions of the detector will produce different amounts of light. In either case, whether the light generated varies or the light collection is not uniform, the detector resolution is degraded.

Surface compensating attempts to remove these variations by changing the reflectivity of the detector's surfaces to give a untform pulse height 
response. The procedure maps the detector by measuring the channel number of the photopeak as a function of position on the detector's surface using a well-collimated $\gamma^{-r a y}$ source. Places on the surface with large pulse helghts are then polished to remove 11ght. The mapping is done again and the process is then iterated until the response is made uniform at the expense of throwing away 1ight.

Surface compensation is usualiy done with a ${ }^{137} \mathrm{Cs}(611 \mathrm{KeV})$ source. In this case, corrections are made only for the light generated and collected from the surface reglons. Recently, higher energy $6 \mathrm{MeV}$ sources $\left({ }^{244} \mathrm{Cm}+{ }^{13} \mathrm{C}\right)$ have been used to probe more deeply into the crystal's volume. This allows the surface compensation to correct for more of the volume irregularities, thereby resulting in better detector resolution ${ }^{3}$. Figure 1 illustrates the technique with uniformity measurements before and after surface compensating. This uniformity map was made for the collimated source placed at one-inch intervals along the length of the detector, and was done at both $661 \mathrm{KeV}$ and 6 MeV. The data was taken by Bicron Corporation during the fabrication of a $26.7 \mathrm{~cm} \times 55.9 \mathrm{~cm}$ long NaI(T1) detector for Boston University ${ }^{4}$. This crystal was made by joining two $26.7 \mathrm{~cm}$ diameter cylinders, one $35.6 \mathrm{~cm}$ long and the other $20.3 \mathrm{~cm}$ long. The optical joint at $20 \mathrm{~cm}$ froin the phototube end is clearly seen in both the $661 \mathrm{KeV}$ and $6 \mathrm{MeV}$ measurements. The surface compensation reduced the $5.2 \%{ }^{137} \mathrm{Cs}$ non-uniformity to 1.67 but only redistributed the $6 \mathrm{MeV}$ non-uniforitty which remained at $2 \%$. However, the desired result was obcained which was to improve the detector's resolution at $6 \mathrm{MeV}$ from 3.5\% to $2.6 \%$. The ${ }^{137}$ Cs resolution was essentially unchanged due to the resolution at low energies being dominated by photo-electron statistics.

The complete Boston Unfversity spectrometer consists of the above described $26.7 \mathrm{~cm} \dot{\mathrm{x}} 55.9 \mathrm{~cm}$ central crystal surrounded by four $\operatorname{NaT}(\mathrm{T} 1)$ 
segments to obtain a $48.3 \times 55.9 \mathrm{~cm}$ detector. Preliminary energy resolution measirements have obtained $2 \%$ for $330 \mathrm{MeV}$ electrons ${ }^{4}$ and $1.6 \%$ for $130 \mathrm{MeV}$ gamma rays. The solid angle over which these data were collected was quite small (a few msr), but, nonetheless, these results are very encouraging.

\section{III. 'Mapping Large Crystals with Cosmic Ray Muons}

At Brookhaven National Laboratory (BNL) we are developing a new technique using high energy muons to probe the intertor of a NaI(T1) crystal and locate internal defects. The muon is the ideal probe, since $\gamma$ rays or electrons create electromagnetic showers which extend over a large volume. In contrast, the heavier muon produces only a narrow well-defined lonization track, and being weakly interacting, avolds nuclear reactions which would smear out its energy loss. The amount of energy deposited depends only weakly upon the incident muon energy and, to first order, is a function only of the thickness of the $\mathrm{NaI}(\mathrm{Tl})$ through which it passes. Therefore, after correcting for differences in NaI path length, the remalning differences in signal amplitudes reflect the nonuniformities within the NaI crystal.

Since we want a technique which can be used to reject and accept ingots early in the fabrication process, we are using the natural background of cosmic ray muons for cur source. This avoids the severe logistic problems in moving large lingots of NaI back and forth hundreds of miles between the crystal manufacturing plant and an accelerator facllity. The procedure involves measuring NaI(TI) spectra which are coincident with detectors definIng narrow trajectorles through the volume of the NaI(T1). These spectra are then fit with Monte Carlo calculated lineshapes which include the effects of the muon energy straggling spectrum and the shape of the NaI(T1) detector. From the fits we obtain the energy shift of each trajectory's experimental lineshape from the Ideal Monte Carlo Ineshape. These energy shifts can then 
be used to determine the overall uniformity of the detector, similar to the Y-ray measurements above, but they can also be used to study defects deep in the interior. In fact, by using established CAT techniques a detailed map or 'Image' of the detector can be reconstructed.

The energy loss spectra of muons in the BNi MkIII $24 \mathrm{~cm} \times 36 \mathrm{~cm}$ NaI(Tl) detector are presented in Fig. 2. The top frame shows the experimental singles spectrum (solid line) and the calculated spectrum (dashed line) produced by cosmic ray muons. The Monte Carlo calculation used the published muon energy spectrum at sea level, the observed $\cos ^{2} \theta$ angular dependence 5 (where $\theta$ is the angle away from the zenith) and the Vavilov energy loss distribution ${ }^{6}$. The difference below $75 \mathrm{MeV}$ is due to the 'soft' component of the cosmic ray background coming from muons showering in the air and the bullding material above the detector. This component produces a tall at lower energles and was not included in the calculation.

The bottom frame of Fig. 2 illustrates the result of limiting the muon tzajectories to a column $6.5 \mathrm{~cm}^{2}$ in cross section which intersects approximately $24 \mathrm{~cm}$ of $\mathrm{NaI}(\mathrm{T} 1)$. The data is plotted with 1 is statistical errors and the solid curve is the Monte Carlo calculation with all the ingredients described above. The width of the energy loss lineshape is dominated mostly by Landau straggling with a small contribution from the broad energy spectrum of the incident muons. Figure 3 shows the Vavilov distributions for muons with $2.5 \mathrm{GeV} / \mathrm{c}$ and $270 \mathrm{MeV} / \mathrm{c}$ momenta traversing $24 \mathrm{~cm}$ of $\mathrm{NaI}(\mathrm{TI})$. The cosmic ray spectrum peaks at $600 \mathrm{MeV} / \mathrm{c}$ and is asymmetric with the mean at much higher energies. A factor of 2 improvement in the resolution of the energy loss peax could in principle be obtained using lower energy, mono-energetic muon beams from an accelerator. However, cosmic-ray muons are nearly minimum lonizing, depositing about $4.8 \mathrm{MeV}$ in each $\mathrm{cm}$ of $\mathrm{NaI}$, so that a minimum muon moinentum of 
$350 \mathrm{MeV} / \mathrm{c}$ is necessary to fully penetrate the $48 \mathrm{~cm}$ of the planned large NaI. Such low energy beams are normally accompanied by appreciable backgrounds. In any case, the logistics involved in repeatedly transporting a large ingot, sealed in a temporary housing to prevent hydration, large distances from the crystal growing faclltty to an accelerator render this option unworkable. Cosmic rays, are almost as good and are avallable everywhere!

Since the hard muon component of the cosmic ray flux is only about .008/ $\mathrm{cm}^{2} / \mathrm{sec} / \mathrm{sterad}$, it is essential to collect data on all trajectories simultaneously. To achieve this we have bullt arrays of plastic scintillators above and below the NaI(T1) that define the desired muon trajectories. Figure 4 is a photograph of the scanning array, which consists of four planes of crossed scintillator bars, two above the detector being studied and two below. These planes define approximately $3.8 \mathrm{~cm} \times 3.8 \mathrm{~cm}$ areas of overlap. A similar arrangement below defines a column of muons which traverse the volume being studied. With 16 bars in each plane, this arrangement allows for $16^{2} \times 16^{2}$ or 65,536 possible trajectories. It is necessary to have the information from a large number of trajectorles to accurately perform the Fourier transforms necessary to create the image of the crystals. The scanning array accepts crystals that are up to $75 \mathrm{~cm}$ in diameter, although the goal of the present effort is a $48 \mathrm{~cm}$ diameter detector. The crystals will be transported from the furnace area on the cart shown in Fig. 4 and w111 slide into the area on a rail system. The 24 cm diameter BNL-MKIII detector is pictured here entering the array.

The data from this array is extremely complex. Let us start with some simple analysis of the preliminary data from the BNL-MKIII NaI(T1). Taking trajectorles lying in planes at right angle to the axis of the detector (see Fig. 5 insert), NaI(Tl) spectra can be generated for $3.8 \mathrm{~cm}$ thick slices from 
one end of the detector to the other. The muons from the vartous trajectorfes that are contained in each slice produce a sheet of light in the detector 3.8 cm thick, whose distance can be varied from the phototube end of the WaI(Tl). The dependence of the peak location of the NaI(T1) spectrum upon the distance from the phototube end is plotted in Fig. 5 (top). This is quite a different: uniformity measurement from that plotted in Fig. 1. In this case one studies the phototube pulse helght for 11 ght produced throughout each $3.8 \mathrm{~cm}$ thick disk, not just from the skin of the detector as before. The results show a $2 \%$ vartation from one end to the other. The large excursion near the tube end may be due to light being lost by total internal reflection when it is produced very close to the phototube windows. In any case, this variation is mucin. larger than what is indicated by map made with a collimated 6 MeV source moved along the outside of the detector parallel to its axis. The largest and smallest $6 \mathrm{MeV}$ varlations are shown in Fig. 5 (bottom). The surface compensation chosen to minimize the variation at $6 \mathrm{MeV}$ is not likely to be the correct one to perform on a high-energy detector. It is interesting to note that the muon and $6 \mathrm{MeV}$ scans of Fig. 5 are almost anti-correlated. Stnce the muon scan here averages over an entire slice, it is much more representative of what will be encountered by a high-energy shower that fills essentially the whole volume of $\mathrm{NaI}(\mathrm{TI})$.

\section{Imaging the NaI Interior}

Beyond averaging over disks perpendicular to the crystal axis, as was done to obtain Fig. 5 (Lop), the next step is to deconvolute the data and obtain a two-dimensional lmage of the uniformity within each disk. There are many ways to do this. We have chosen a standard known as Ramachandran's Algorithm ${ }^{7}$. If we choose an $x-y$ coordinate system within each disk and let L represent a muon trajectory, whose normal through the origin is of length $r$ 
and makes an angle $\theta$ with the positive $x$-axis, then each trajectory is specified by the line $L(r, \theta)$ whose equation is

$$
r=x \cos \theta+y \sin \theta
$$

The energy loss of a muon following this trajectory is

$$
\Delta E_{L}=\int \frac{d E}{d s} d s,
$$

which, including all effects, is determined by the Monte Carlo calculation described in the previous section. This indeed reproduces the data, as demonstrated in fig. 2, except for shifts due to non-uniformities. An expression similar to (2a) can be written for the actual signal amplitude AL produced by the muon following the trajectory $\mathrm{L}$,

$$
A_{L}=\int \frac{d A}{d s} d s
$$

With the photomultiplier signals calibrated so that (2b) and (2a) have the same scale, the deviation in the signal produced by a muon from what would be expected of a trajectory containing perfectly uniform material is

$$
T(L(r, \theta))=A_{L}-\Delta r_{L}=\int_{L} f(x, y) d s,
$$


where $f(x, y)$ is the local deviation from uniformity. It is $T$ that is known for $z$ large number of trajectories, and $f$ is the uniformity distribution that we wish to deduce. It is straightforward to show that $f$ is given by ${ }^{8}$

$$
f(x, y)=\frac{1}{2 \pi} \int_{0}^{\pi} d \theta \int_{-\infty}^{\infty} T(R, \theta) \phi(r-R) d R,
$$

where $\phi$ is a function whose Fourier transform is just

$$
\phi(\omega)=|\omega| \quad, \quad \text { for } \operatorname{smali} \omega .
$$

In this algorlthm, all of the trajectories within a disk are broken up into classes, each composed of parallel lines at a flxed $\theta$, so that within one class $R=k a$ where $k=0, \pm 1, \pm 2, \ldots$ A sum over $k$ for each class, and a sum over all classes then replaces the Integration over R. The array of Fig. 4 covers approximately $90^{\circ}$ in $\theta$, so that two sets of measurements are necessary in order to complete the angle integration, the second with the detector rotated $90^{\circ}$ relative to the first.

The power in this method comes from the abllity to introduce the weighting function $\Phi$ which can be used to correct for spurious effects that arise from using a finite rather than an infinite number of trajectories. Specifically, we use

$$
\begin{aligned}
& \phi(0)=\frac{4}{\pi a^{2}}, \\
& \phi(k a)=-\frac{4}{\pi a^{2}\left(4 k^{2}-1\right)} \quad, \quad k= \pm 1, \pm 2, \ldots
\end{aligned}
$$


so that $\phi(r-R)$ peaks when $r$ is within the $3.8 \mathrm{~cm}^{2}$ column of the trafectory at $R$ and has small negative calls due to neighboring trajectorles. This algorithm has been shown to yield a reconstruction of $f(x, y)$ that is machematically exact within the region of interest. (the disk of NaI in our case), but oscillates infinitely outside of this volume ${ }^{8}$.

We have tested this algorithm with different shaped pleces of plastic scintillator placed at differste Neglecting variations within the scintillators, the algorlthm has correctly located the positions, orientations, and sizes of these objects.

\section{Results from a Prototype Array}

The $24 \mathrm{~cm} \times 36 \mathrm{~cm}$ BNL-MKIII is presently in the scanning array of Fig. 3 collecting data. The first preliminary analysis ylelded the data shown at the top of Fig. 5. The analysis of the full data set is underway and the results will be presented in a future publication. However, a general flavor of what is to be expected is already available from limited data taken with a prototype array.

The first three-dimensional maps of the interior nonuniformities are shown in Fig. 6. The perspective drawing of the HK-III detector in the lower right-hand corner Indicates the locations of the three orthogonal slices lilustrated above and to the left. The slices are $1 \mathrm{~cm}$ thick and the plots display the uniformity distribution function $f$ using the grey scale to the upper right. The darker tones indicate more negative $f$ due to less pulse helght response ( $\operatorname{small} \mathrm{A}_{\mathrm{L}}$ ) from these regions.

The slice perpendicular to the axis of the detector, shown in the upper left, clearly Indicates a poor region of the NaI(T1). One general problem area is near $\mathrm{Z}=6 \mathrm{~cm}$ and $\mathrm{X}=-7 \mathrm{~cm}$, and produces deviations of roughly 3 to $4 \mathrm{MeV}$ for trajectortes which traverse this section of the detector. Since the 
average energy loss of a trajectory through $25 \mathrm{cru}$ of $\mathrm{NaI}$ is $121 \mathrm{MeV}$, this amounts to a 3\% nonuniformity when averaged along the $25 \mathrm{~cm}$ length of a trajectory. However, because the CAT-scan map has localized the malformation to be in a region only about $5 \mathrm{~cm}$ across, the percentage nonuniformity within this $5 \mathrm{~cm}^{3}$ volume is much larger. In fact, it is roughly $25 / 5 * 3 \%$ or a $15 \%$ reduction in response relative to the rest of the detector. This will have a very large effect on the detector's resolution for $\gamma$ rays.

Figure 6 can be more fully understood by making a comparison with the top frame of Fig. 5. The average uniformity graphed in Fig. 5 can be obtained from the Fig. 6 map by averaging over the disks formed by $3.8 \mathrm{~cm}$ thick slices perpendicular to the detector's axis. The upper right plot in Fig. $6(4 \mathrm{~cm}<$ $\mathrm{z}<5 \mathrm{~cm}$ ) shows the uniformity distribution for a longitudinal olice along the NaI(T1)'s $35 \mathrm{~cm}$ length. The phototube end is at the right of the map. Averaging over the $x$-dimension with bins of $\Delta y=3.8 \mathrm{~cm}$ yields the same getieral features of Fig. 5. That is, the response of the detector is lower (darker) at the end opposite the phototubes and gradually increases (1ightens) in moving toward the phototubes. Whereas, the map in Fig. 6 is incomplete, there is evidence for general darkening again less than $10 \mathrm{~cm}$ from the phototubes.

The most interesting aspect of CAT scan maps is that they provide the location and values of crystal and light collection nonursformities which can be used with an electromagnetj.c shower code to produce realistic predictions of the detector's performance. This can be done by using the uniformity map to modify the result of the Monce Carlo shower calculation at each volume element. The advantage of this combined calculation 18 that the map empirically allows the inclusion of the largest unknowns in any shower calculation, the imperfections in the crystal, and the variation in light collection. Because of the technique used, it ircludes all of the effects of 
detector nonunfformities discussed in the introduction. The ultimate goal of such a calculation would be to accurately predict the detector's performance for $\gamma$-ray detection early in the fatrication process at the factory. The economic advantages are obvious since this technique could reject or accept ingots before undergoing the costly and lengthy surface compensation procedure. Work is currently in progress developing this procedure.

VI. Conclusion

The cosmic-ray muon scanning array provides information on NaI(T1) crystalshusing some 65,536 trajectories, each measuring the NaI(T1) response tc high energy muons. Hith this information it is possible to use established computer-aided-toinography techniques to deconvolute these integrated responses and froduce a detalled picture of the detector's interior: The interesting feature of this detailed map is that it is the first realistic measurement of the detector's overall response to energy deposited in a localfzed volume of the $\mathrm{NaI}$ and it is just this information of crystal nonuniformities and light collection nonuniformities which is lacking in any fundamental calculation of the $\mathrm{NaI}(\mathrm{T} 1)$ response function to $\gamma$ rays. Therefore, the results of this map could be used with an electromagnetic shower code to accurately predict the detector's performance when detecting high energy $\gamma$ rays.

The system is presently being tested using the BNL MK-III $25 \mathrm{~cm} \times 35 \mathrm{~cm}$ $\mathrm{NaI}(\mathrm{TI})$ and soon thereafter the apparatus will be moved to Bicron Corp. for selerting and compensating the LEGS $48 \mathrm{~cm} \times 48 \mathrm{~cm} N a I(T 1)$ crystal.

Acknowledgement

We gratefully acknowledge the close cooperation of the staff of Bicron Corporation who are participating in this eifort through the DOE Industrial Technology Exchange Program. 


\section{References}

1. A. M. Sandorfi, M. J. LeVine, C. E. Thorn, G. Glordano, G. Matone, and C. Schaerf, IEEE Transaction on Nucl. Sci., Vol. NS-30, No. 4, 3083 (1983).

2. D. H. Dowe11, H. P. Zlegler, A. M. Sandorfi, B. Ziegler, and B. Schock, "The Response of a $24 \mathrm{~cm} \times 36 \mathrm{~cm} \mathrm{NaI}(\mathrm{T} 1)$ Spectrometer to $\gamma$-Rays Between 50 and $300 \mathrm{MeV} "$, NIM (In press).

3. A. M. Sandorfi and M. T. Collins, NIM $\underline{222}$ (1984) 479.

4. J. Miller, private comunication.

5. B. Rossi, "Interpretation of Cosmic Ray Phenomena", Reviews of Modern Physics 20 (1948) 537; J. E. Hooper and M. Scharff, The Cosmic Radiation (Methven and Coc L.td., London, 1958), p. 151-55.

6. S. M. Seltzer and M. J. Berger, "Energy-Loss Straggling of Protons and Mesons: Tabulation of the Vavilov Distribution"; B. J. Fineman, "Probing the Interior of a 19" $\times 19^{\prime \prime}$ NaI(T1) Crystal with Cosmic Ray Muons--A Monce Carlo Simulation," LEGS Report No. 8.

7. G. Ramachandran and A. Lakshminarayanan, Proc. Nat. Açad. of Sc1. U.5. 68, 2236 (1971).

8. L. A. Shepp and B. F. Logan, IEEE NS-21, 21 (1974). 
Figure 1. Uniformity measurements for the Boston University NaI(TL) central derector made with ${ }^{137} \mathrm{Cs}(661 \mathrm{keV})$ and ${ }^{244} \mathrm{Ca}+{ }^{13} \mathrm{C}$ (6 MeV) gamina-ray sources. The top frane shows measurements before surface compensation and the lower frame illustrates the unfformity after surface compensation. The photopeak channel numbers are relative and the gains have been changed between measurements.

Figure 2. Comparison of $\mathrm{NaI}(\mathrm{T} 1)$ spectra with Monte Carlo calculations for singles and colncidence data. See text for detalls.

Figure 3. Vavilov energy loss distributions for mono-energetic muons traversing $25 \mathrm{~cm}$ of $\mathrm{NaI}(\mathrm{Tl})$. These momenta, $2.5 \mathrm{GeV} / \mathrm{c}$ and $270 \mathrm{MeV} / \mathrm{c}$, are at the high and low half-heights of the cosmic-ray muon spectrum at sea level.

Pigure 4. Photograph of the cosmic-ray muon CAT scanner. The Brookhaven MK-III 25 cu $\times 35 \mathrm{~cm} \mathrm{NaI}(\mathrm{T} 1)$ detctor is shown entering the scanning array. NaI(TI) detectors are rolled into this scanner on rails which can be attached to the movable transfer cart shown at the lower right.

Figure 5, Tog. Average uniformity of the BNL-MK III NaI(T1) as a function of distance from the photomultipler tube end of the detector. The average uniformity is the detector response to cosmic-ray muons integrated over $3.8 \mathrm{~cm}$ thick slices as a function of distance along the detector. 
Figure 5. Botton. $6.1 \mathrm{MeV}$ uniformity measurements of the BNL-MK III detector also taken along the length of the detector. The best and worst data are the smallest and largest variations in pulse helght for paths along the sides of the detector but at different positions around the detector's circumference.

Figure 6. Three-dimensional image of the response of the BNL-MK III NaI(TI) to minimun lonizing, cosmic-ray muons. The image was made using data from a prototype scanning array and standard computer-aided-comography techniques. The locations of the three orthogonal slices, each one centimeter thick, are indicated in the drawing at the lower right. The grey scale gives the energy shift of the rusons away from the expected energy loss as given by a Monte Carlo calculation. 
BU $26.7 \mathrm{~cm}$ Dia. $\times 55.9 \mathrm{~cm}$ LONG NaI (T

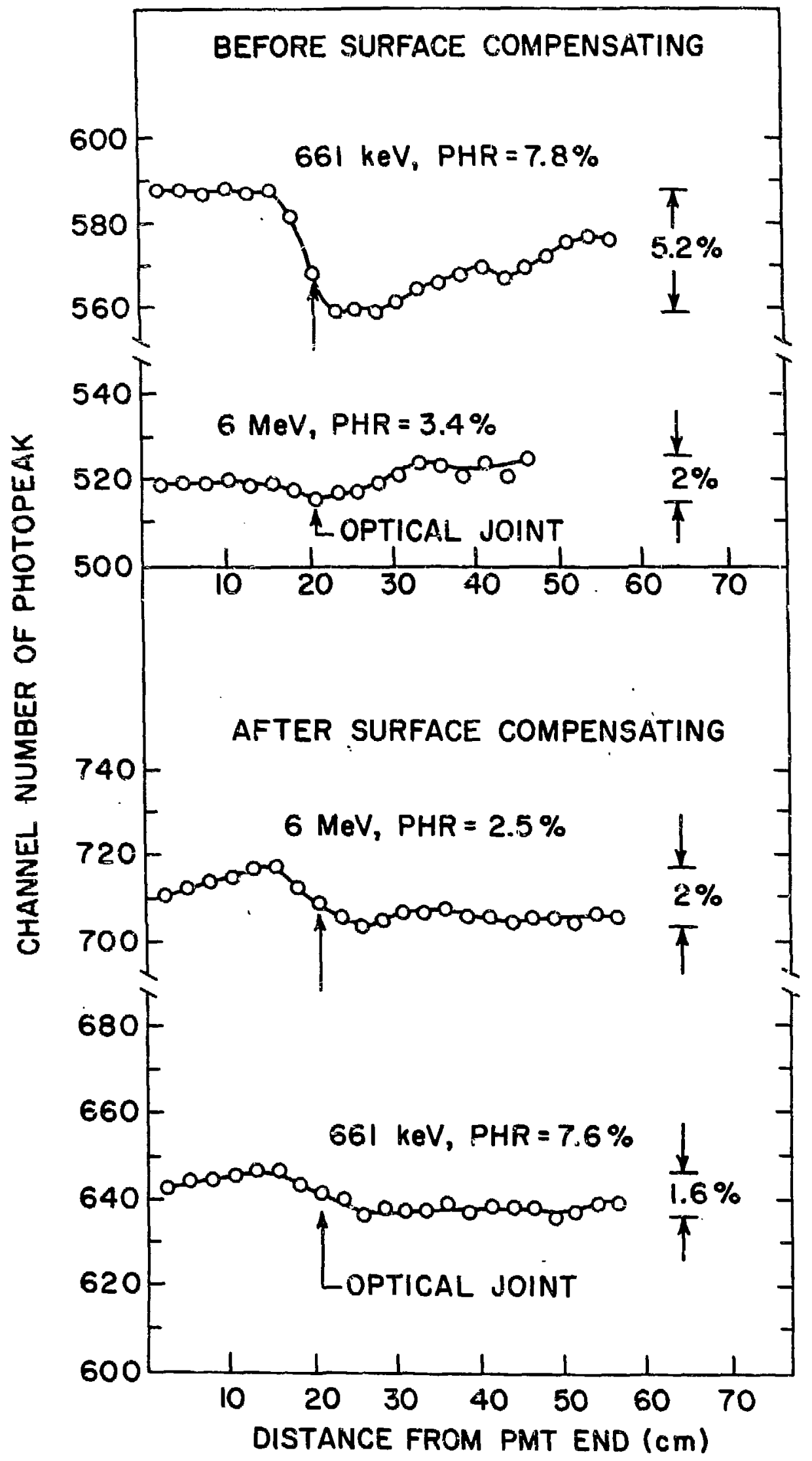


BNL $24 \mathrm{~cm}$ Dia. $\times 36 \mathrm{~cm}$ LONG Na I $(T l)$

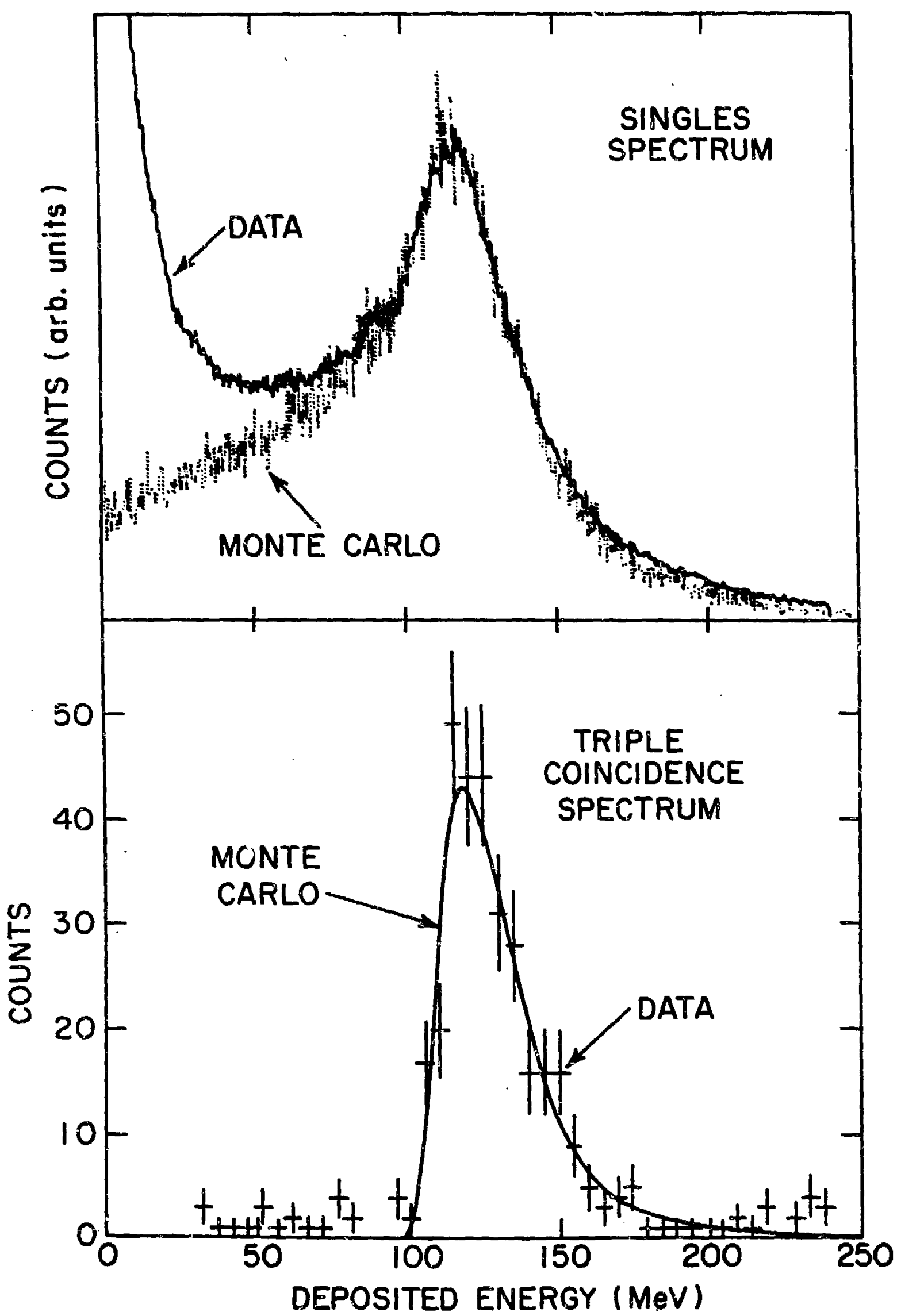


VAVILOV DISTRIBUTIONS

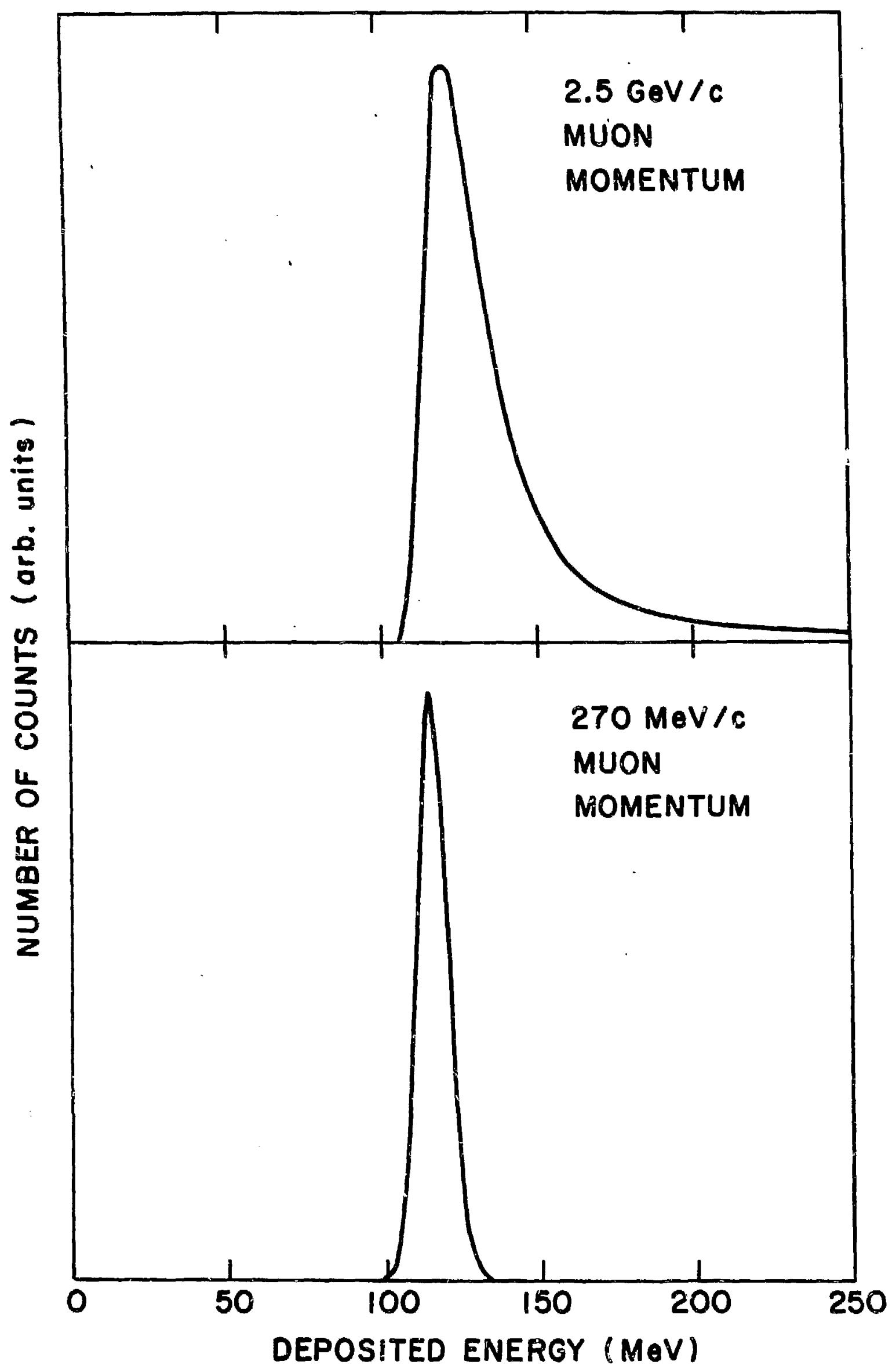




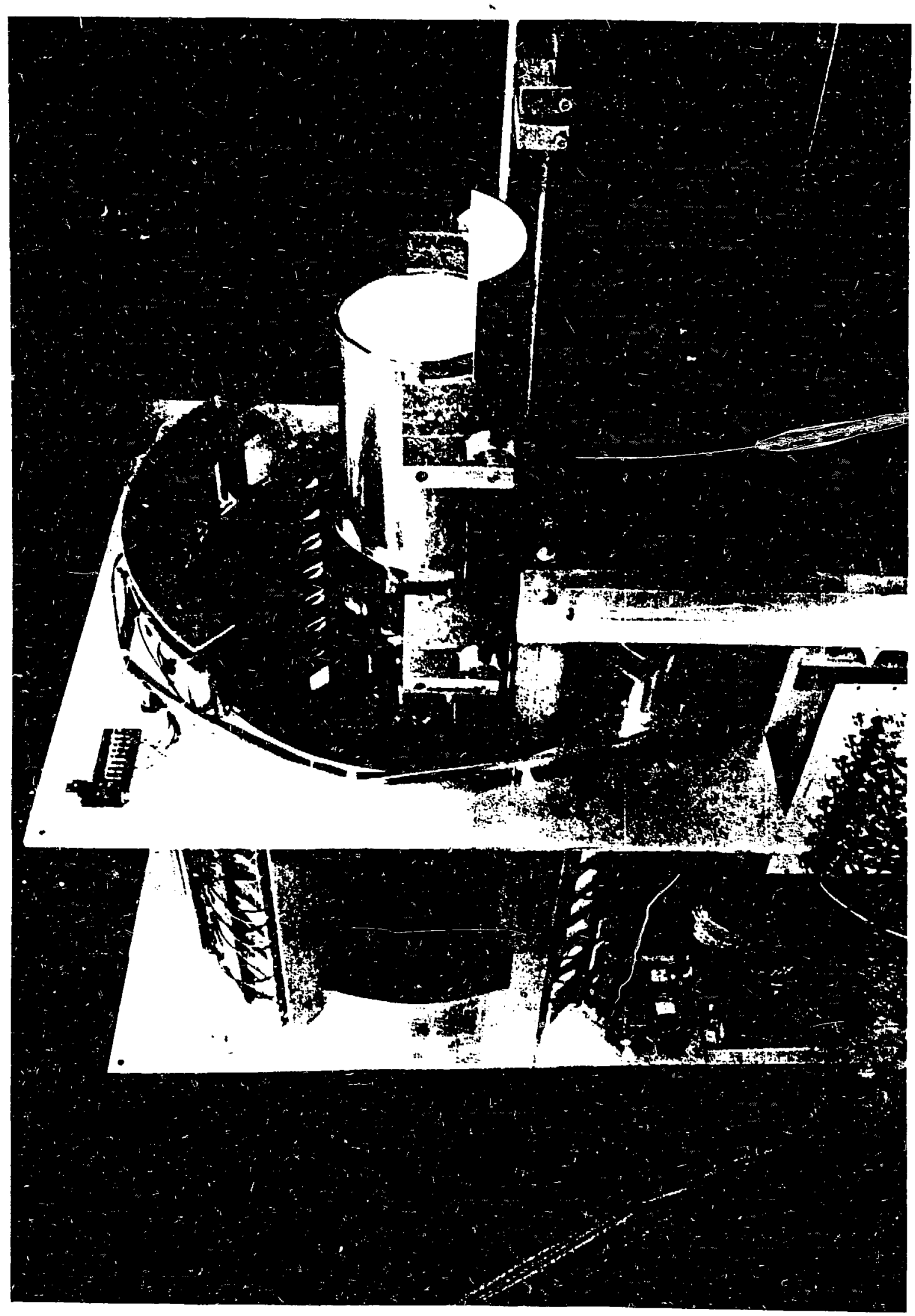



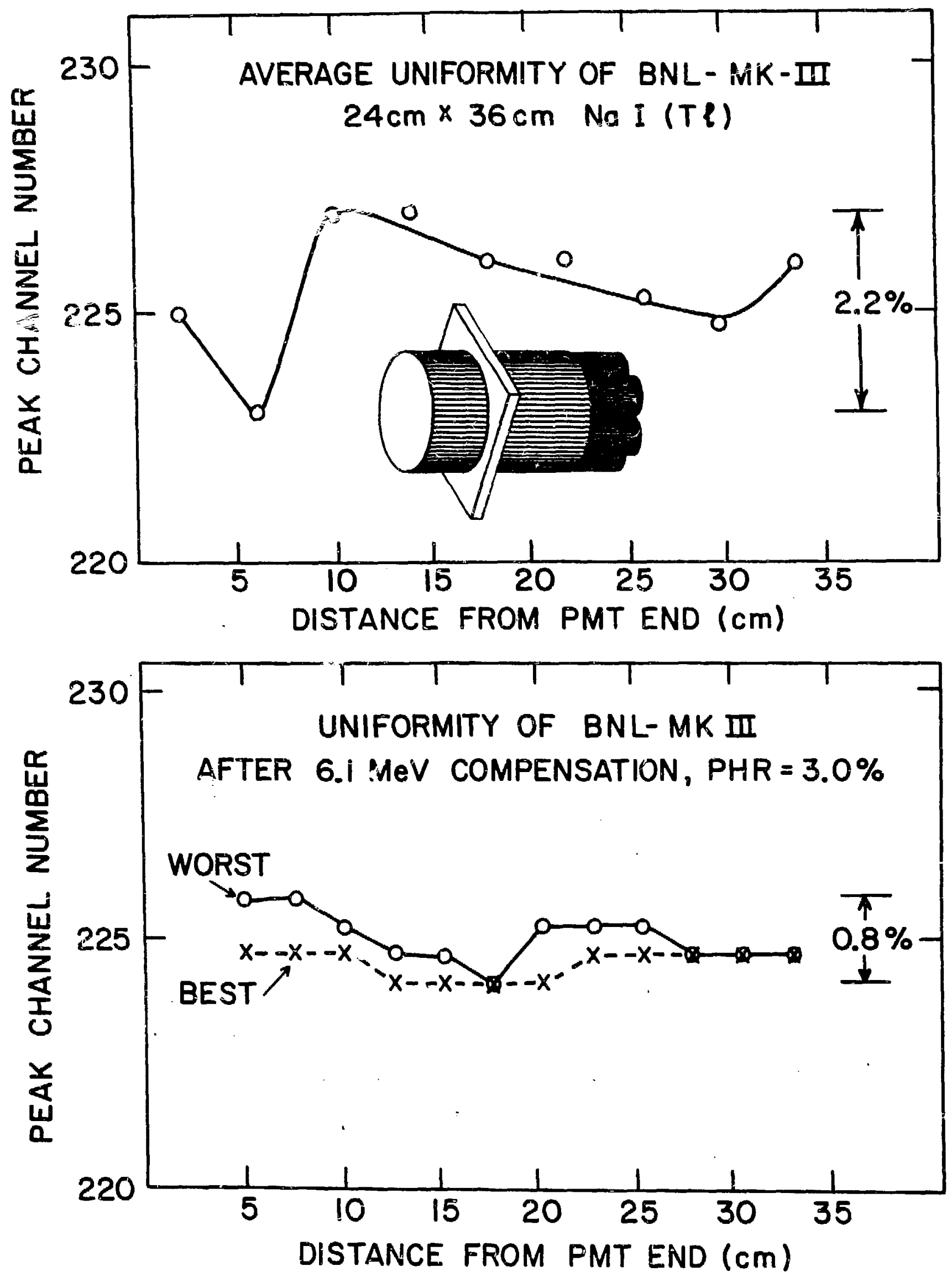

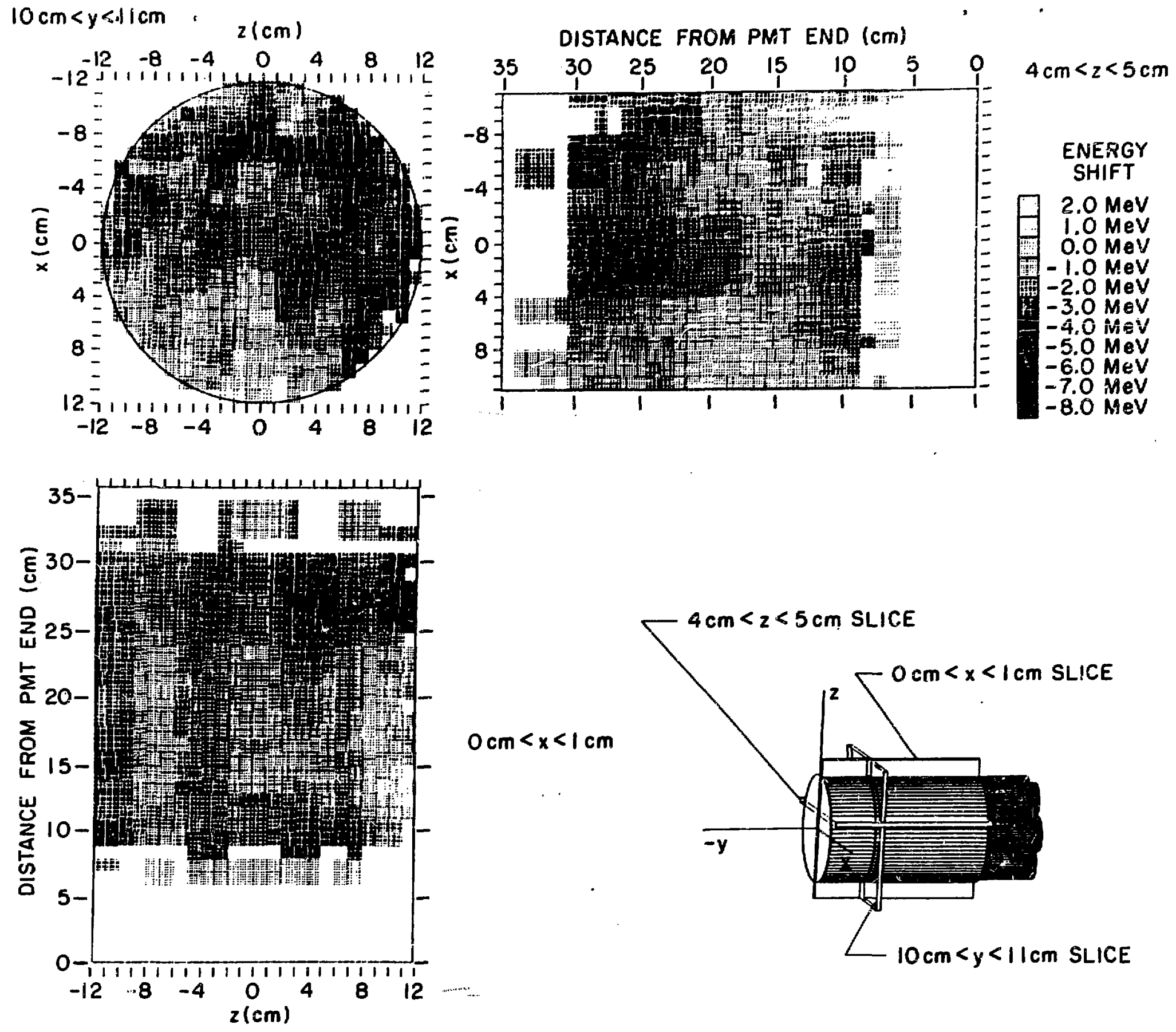